\title{
La Pareja Pedagógica en el Ámbito Universitario, un Aporte a la Didáctica Colaborativa
}

\author{
Diana G. Bekerman ${ }^{(1,2)}$ y Laura A Dankner ${ }^{(2)}$ \\ (1) Grupo de Investigación en Aprendizaje y Didáctica de la Ciencias Naturales y Química \\ (GIADiCieNQ, Asociación Química Argentina). \\ (2) Cátedra de Química Orgánica II, Departamento de Química Orgánica, Facultad de Farmacia y \\ Bioquímica, UBA, Junín 956, (1113) Buenos Aires-Argentina (e-mail: \\ dianabekerman@gmail.com)
}

Recibido Nov. 10, 2010; Aceptado Nov. 22, 2010; Versión final recibida Dic. 14, 2010

\section{Resumen}

El objetivo de este trabajo fue implementar la modalidad pareja pedagógica en el dictado de la asignatura Química Orgánica II en la Universidad de Buenos Aires-Argentina, a modo de experiencia en el ámbito de la enseñanza universitaria. Para ello, se adaptaron estrategias utilizadas en otros niveles educativos, en especial en lo concerniente a respetar los estilos de trabajo de las docentes implicadas. Se desarrolló un sitio Web exclusivo para este grupo, con materiales didácticos especialmente diseñados. Todos los actores implicados, profesoras y alumnos, mostraron un alto grado de satisfacción con esta nueva estrategia didáctica, por lo que amerita ser continuada y ampliada a otros cursos universitarios.

Palabras clave: pareja pedagógica, estilos de aprendizaje, didáctica colaborativa, enseñanza

\section{The Teaching Couple at University Level, a Contribution to Collaborative Teaching}

\begin{abstract}
The aim of this study was to implement the teaching couple method for the Organic Chemistry II course at the University of Buenos Aires-Argentina as an experience in university teaching. To do this, strategies used at other educational levels, in particular, preserving the learning styles of the instructors involved, were adapted. A Web site fully dedicated to the teaching group including all teaching material was specially designed. All people involved, instructors and students, showed high degree of satisfaction with this new teaching strategy, so the method should be continued and implemented in other university courses.
\end{abstract}

Keywords: teaching couple, learning styles, collaborative teaching, education 


\section{INTRODUCCIÓN}

La Facultad de Farmacia y Bioquímica de la UBA ha introducido recientemente cambios en la asignatura Química Orgánica II en función del nuevo Plan de Estudios. Estos cambios implicaron un recorte y reordenamiento de contenidos. Esta modificación requirió una readaptación didáctica en el dictado de dicha asignatura. Al implementar la tarea se tuvo en cuenta una serie de consejos que plantean Benoit y Haugh (2001) para establecer una práctica eficaz en la "enseñanza cooperativa" con respecto a los siguientes temas: i) planificación; ii) roles individuales; iii) estrategias en el aula y iv) evaluación de la clase y del alumnado. Como todo aprendizaje requiere un análisis de las partes que componen un determinado conocimiento, se hace legítimo e indispensable conducir a los alumnos hacia un análisis diferenciador originado en el criterio complementario de ambas docentes.

El contenido es un objeto simbólico y las características del mensaje dependerán del modo en que es organizado y transmitido. Como todo mensaje, el contenido transmitido está sujeto a interpretación y modificación. Cuando los docentes se proponen transmitir determinados contenidos, se produce otra modificación de los saberes ya que, nuevamente, se produce una selección y adecuación del mensaje. Con el fin de facilitar su comprensión por parte de los alumnos, los docentes utilizamos simplificaciones, analogías, ejemplos, aplicaciones; cada docente da más importancia a ciertos elementos que a otros. Se produce así una nueva transformación con el fin de generar una representación útil y accesible a los alumnos. El contenido enseñado (currículo real), deriva de las influencias del currículum, de la bibliografía y, cómo ya fue mencionado, de las perspectivas de quienes participan en su elaboración y transmisión (Feldman y Palamidessi, 2001). Esto plantea la necesidad de tener en un mismo curso dos docentes que aborden los temas desde diferentes puntos de vista, utilizando diferentes lenguajes, ejemplos y aplicaciones, previamente consensuados entre ambos, para transmitir los contenidos, transformándose así en una tarea colaborativa. Sin embargo, el uso de estas metodologías de enseñanza cooperativa presenta las reticencias derivadas de la dificultad del trabajo en grupo, y requiere del profesorado un amplio conocimiento de la materia, tiempo para la preparación del material y la corrección del trabajo entregado por los alumnos, e incrementa considerablemente la atención proporcionada al alumnado con relación a las tutorías contempladas en la enseñanza tradicional (Menéndez et al, 2009).

La enseñanza centrada en el aprendizaje considera que cada persona aprende de manera diferente y posee un potencial, conocimientos y experiencias distintas, es decir, existen diversos estilos de aprendizaje, a partir de los cuales procesamos la información recibida del medio y la transformamos en conocimiento. Aunque las estrategias varían según lo que se quiera aprender, cada uno tiende a desarrollar ciertas preferencias o tendencias globales, tendencias que definen un estilo de aprendizaje. Son los rasgos cognitivos, afectivos y fisiológicos que sirven como indicadores relativamente estables de cómo los alumnos perciben interacciones y responden a sus ambientes de aprendizaje, es decir, tienen que ver con la forma en que los estudiantes estructuran los contenidos, forman y utilizan conceptos, interpretan la información, resuelven los problemas, seleccionan medios de representación (visual, auditivo, o kinestésico, entre otros).

A modo experimental se implementó la planificación de dicho dictado, utilizando la modalidad de pareja pedagógica ampliamente difundida en los niveles inicial, primario y secundario (Pombo y Costa, 2009; Zhang y Yan-Hua, 2008; Ribas y Vital dos Santos, 2010). Se entiende por pareja pedagógica al trabajo en equipo que realizan dos docentes sobre el abordaje pedagógico y didáctico en relación a un grupo de alumnos. Desde el momento de la planificación, puesta en acción o en ejecución, elaboración de materiales y momento de reflexión, como así también el planteamiento y elaboración de intervenciones compartidas. Esta implementación se fundó en los siguientes hechos: i) Las docentes implicadas tienen diferentes estilos de enseñanza, utilizando la escala de Felder y Silverman (1988), lo cual permitiría a los estudiantes contar con dos perspectivas diferentes en la presentación de materiales didácticos (Suárez et al, 2003). Para los docentes, descubrir su propio estilo de aprendizaje, haciendo conscientes los procesos cognitivos propios, para ampliar sus horizontes conceptuales metodológicos y principalmente didácticos, sería una forma de apoyo al proceso de autoconocimiento en los estudiantes que tienen bajo su 
responsabilidad; ii) La articulación de la enseñanza a través de "parejas pedagógicas" ha resultado muy exitosa en otros niveles educativos ya que promueve una actitud colaborativa entre pares (peer coaching) y brinda a los docentes la oportunidad de recibir retroalimentación antes de presentar la clase; iii) Es indispensable reconocer la existencia de una pluralidad de lenguajes (Bekerman y Galagovsky, 2009) en la comunicación de la Química, y el consenso y la diversificación deben estar pautadas a fin de aprovechar las potencialidades individuales; iv) La implementación de un sitio Web, alojado en un servidor gratuito tiene las ventajas de permitir una accesibilidad amigable y compartida, de rápida llegada a los estudiantes, en cualquier momento y lugar; y v) Es indudable que las tareas colaborativas en el trabajo del Farmacéutico y del Bioquímico son indispensables, tomando en cuenta que en el ámbito profesional se impulsan las interconsultas con otros especialistas, las discusiones en foros y los debates; así como también el trabajo en grupo que promueven algunas empresas entonces es menester comenzar a ejercitar su implementación en las aulas universitarias. Si se valora como tal el aprendizaje colaborativo (Delors, 1997), la enseñanza colaborativa debería ser prioritaria.

El objetivo de este trabajo consistió en articular dinámicamente la información preparada y discutida por las autoras en un modo colaborativo, presentarla amigablemente en formato cibernético, y coordinar activamente las intervenciones que efectuaron los estudiantes inducidos por los contenidos planteados entre ambas. Esta implementación colaborativa propone restaurar en la enseñanza las fases de integración y de fijación del contenido.

\section{MÉTODOLOGÍA}

La actual asignatura Química Orgánica II cuenta con seis sesiones de trabajos prácticos, doce seminarios de temas teóricos y tres talleres de resolución de problemas. Se instrumentó entre las docentes un calendario de reuniones a fin de decidir las acciones a seguir en cuanto a selección, secuencia y evaluación de contenidos, en arreglo a los contenidos mínimos propuestos por la Cátedra. Se actuó siguiendo los pasos sugeridos por Calzadilla (2001) para producir una tarea colaborativa: 1) estudio pormenorizado de capacidades, deficiencias y posibilidades de los miembros del equipo; 2) establecimiento de metas conjuntas, que incorporen las metas individuales; 3) elaboración de un plan de acción, con responsabilidades específicas y encuentros para la evaluación del proceso; 4) chequeo permanente del progreso del equipo, a nivel individual y grupal; y 5) cuidado de las relaciones socio afectivas, a partir del sentido de pertenencia, respeto mutuo y la solidaridad, y discusiones progresivas, en torno al producto final. Se implementó para el curso compartido de 15 estudiantes un sitio Web (www.quimicuba.com.ar) en el cual se fueron volcando presentaciones PowerPoint elaboradas especialmente para el seguimiento del curso, notas y otras informaciones administrativas propias del curso en cuestión. Cuatro de las clases fueron observadas por la otra docente. Se relevaron los comentarios de los estudiantes y sus mensajes electrónicos.

Atendiendo a los pasos recomendados por Calzadilla (2001) se procedió en primer término a efectuar tests de "estilos" a las docentes con el objeto de encontrar semejanzas o diferencias aprovechables en la implementación del trabajo colaborativo. Se encontraron dos tendencias diferentes en los resultados del Inventario de Felder y Silverman (1988) para las docentes intervinientes. Una de ellas contaba con un predominio visual e intuitivo, la otra tenía predominio verbal y sensorial. Se tuvieron en cuenta estos aspectos en la elaboración del material audiovisual que luego se les presentó a los estudiantes. La inclusión del sitio Web fue muy bien recibida por los 15 estudiantes del Curso y permitió que trajeran a clase las imágenes impresas, lo cual facilitó el seguimiento de las explicaciones verbales. Esta actividad no representa ninguna novedad en el campo de la didáctica. Lo que es nuevo, es la actitud con que en nuestros días son encaradas y revalorizadas. Constituye un complemento indispensable e integrador en el plan general de educación y en la vida de cualquier centro de enseñanza. También pretende ser un correctivo eficaz contra los excesos de la enseñanza teórica, libresca y formal que prevalece en la mayoría de las instituciones. Realmente este tipo de desarrollo cuando está bien organizado y dirigido, ofrece una nueva veta de posibilidades educativas para los estudiantes abriéndoles nuevos horizontes de conocimientos y nuevas perspectivas de acción. 
En esta Sociedad de la Información, es posible transmitir datos a altísima velocidad, elaborar entornos multimediales e interaccionar con personas a distancia, por lo que cobra una relevancia inusitada en la educación superior (Cukierman et al, 2009). Los profesores desempeñan una función de evaluadores y selectores de información adaptada a sus estudiantes, es decir, son un soporte de información y de acceso a recursos para los propios estudiantes. Esta cuestión, fundada en la diversidad de estilos de aprendizaje, permite la complementación y favorece la accesibilidad. El sitio se diseñó con sólo tres páginas Web para acotar la navegación por parte de los estudiantes y evitar distractores: la página principal o de bienvenida, una página con archivos para bajar y otra con sitios recomendados. En la página principal se colocó la dirección electrónica donde los estudiantes pudieron comunicarse con sus docentes. La fluida comunicación a través de los correos electrónicos permitió una evaluación en tiempo real de las dificultades de los estudiantes, y una inmediata discusión entre las docentes a fin de redirigir las estrategias planteadas.

La existencia de documentación escrita y compartida en cuanto a las consultas de los estudiantes, es una ventaja adicional que otorga el trabajo informatizado. En la página de archivos se colocaron todos los materiales escritos y gráficos que se utilizaron para las clases: diez presentaciones en pantalla especialmente elaboradas para este curso, y cuatro archivos que contenían tablas y resultados de ejercitación preparada especialmente. A medida que se obtenían preguntas o comentarios de los estudiantes, algunos textos fueron modificados en coherencia con ellos. La página de sitios recomendados sólo redirigía a sitios con utilidades específicas para el curso (programas para escribir y nombrar fórmulas químicas, sitios para simular espectros, sitios para búsqueda de propiedades físicas de compuestos). Es decir, este sitio funcionó como sitio privado, exclusivo para los estudiantes de este curso particular.

\section{RESULTADOS}

El hecho de trabajar en pareja pedagógica permitió que cada docente pudiera aportar su mejor perfil a los fines didácticos. Los estudiantes de esta Facultad completaron una encuesta con 21 preguntas referidas a 4 temas del dictado de los cursos, calificando cada pregunta con una escala numérica de 1 a 10. La encuesta cumple con la Res. CS 1648/91 y con las Res. CD 738/92 y CD 1243/09. Los 4 temas encuestados son: el curso en general, las clases teóricas, los seminarios (tareas de aula) y los trabajos prácticos de laboratorio. Se incluye en la encuesta la autoevaluación de los estudiantes. Esta metodología, permite tratar de forma cuantitativa y estadística la Calidad Educativa de los cursos, posibilitando el uso del método científico para mejorar la gestión educativa y el desempeño académico de los estudiantes. Todos los estudiantes del grupo enseñado con la modalidad colaborativa se manifestaron conformes frente a la solvencia y coherencia mostradas por sus docentes, a diferencia de otras situaciones planteadas en años anteriores a través de las encuestas llevadas a cabo por la Facultad.

La previsión de reuniones periódicas con fines específicos resultó una herramienta indispensable para la correcta consecución de los objetivos planteados. Estas reuniones se efectuaron en forma presencial o a través de comunicaciones telefónicas frente a las respectivas computadoras. El trabajo directo sobre los textos e imágenes a presentar a los estudiantes mostró una importante incidencia de los estilos de los docentes. Ante determinados conceptos a enseñar con alto componente visual, como por ejemplo la estereoquímica de las reacciones o la química de los hidratos de carbono, se mostró más a gusto la docente con mayor estilo visual (Fig. 1). Esta docente remarca los señalamientos con colores distintos o texturas. La necesidad de presentar más explicaciones verbales en la enseñanza de conceptos, tales como la regioselectividad de reacciones sobre heterociclos o la espectrofotometría de masa, fue abordada con más entusiasmo por la docente con mayor componente verbal en su estilo (Fig. 2). En este caso, la docente recurrió a explicaciones verbales escritas, no a referentes visuales. Las docentes implicadas en la experiencia manifestaron la percepción de un enriquecimiento mutuo, tanto académica como emocionalmente a los fines de la tarea docente. Ante un cambio de programas y de modalidades, muchas veces aparecen dudas en la implementación de las clases, en cuanto a estrategias didácticas, que a nivel universitario no siempre son fáciles de despejar. La posibilidad de intercambio en este tipo de trabajo fue muy efectiva. 


\section{Proyecciones de Fischer, Haworth y Conformacional.}
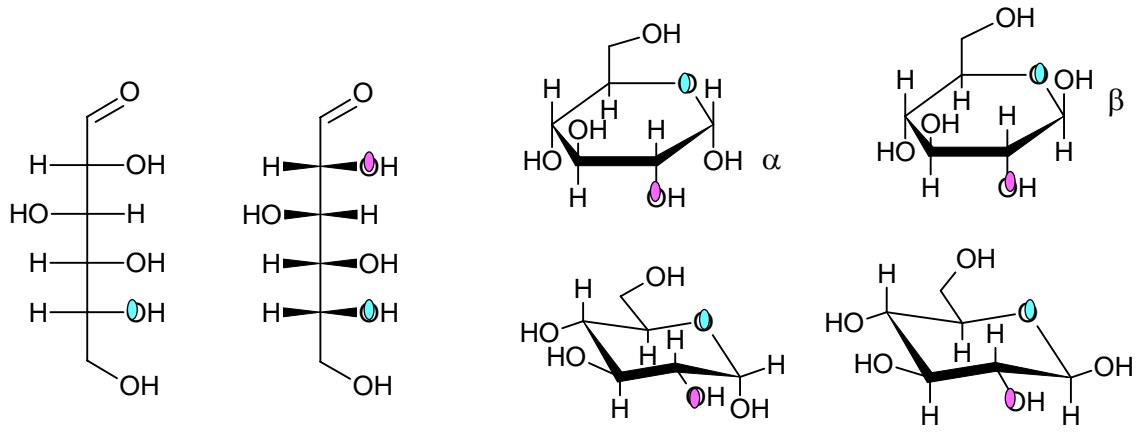

Fig. 1: Ejemplo de pantalla elaborada por la docente con predominancia de estilo visual

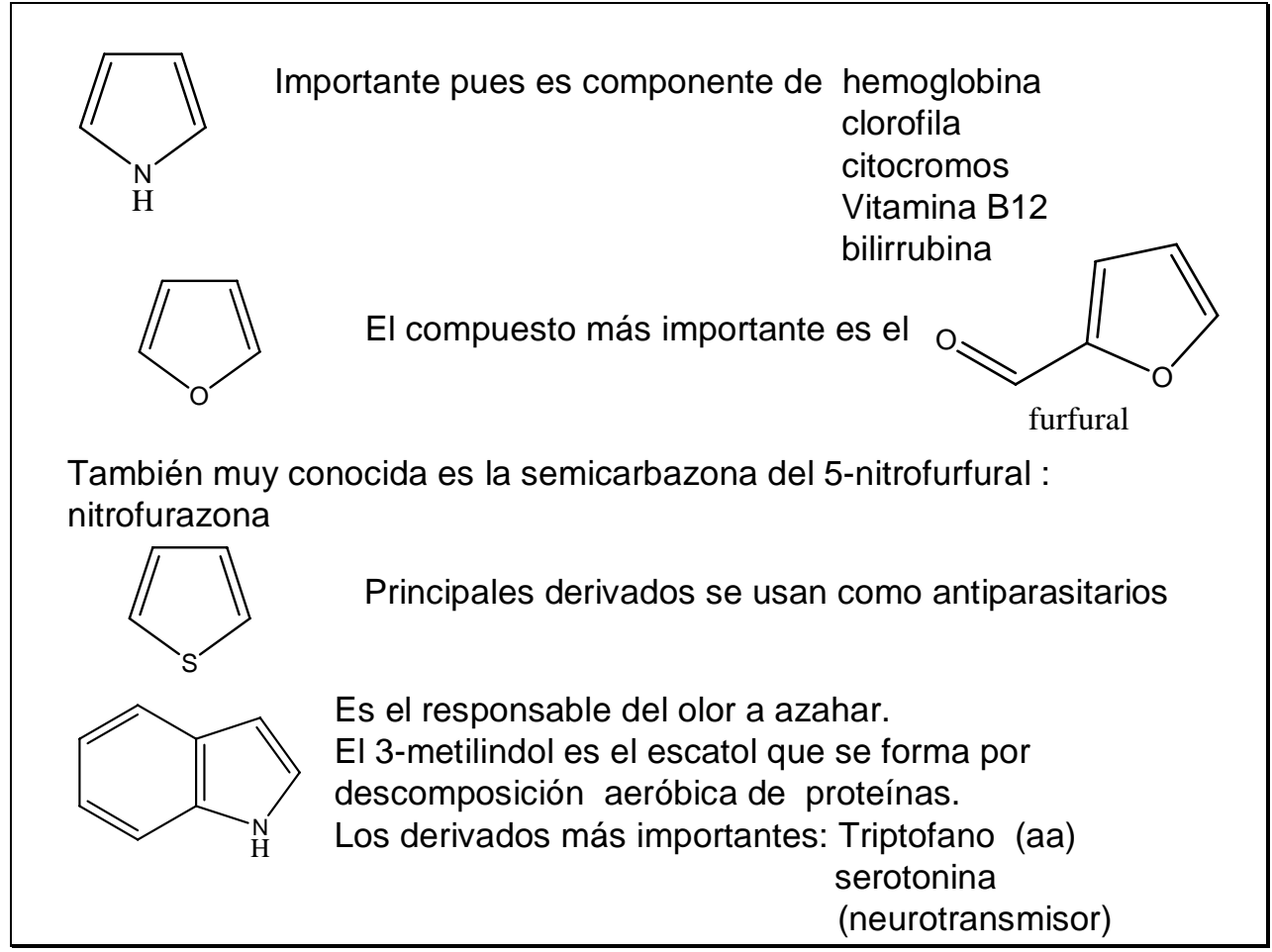

Fig. 2: Ejemplo de pantalla elaborada por la docente con predominio de estilo verbal

\section{CONCLUSIONES}

La responsable implementación del trabajo didáctico a través de "parejas pedagógicas" puede ser una muy interesante opción para mejorar la enseñanza de la Química, en virtud de respetar la diversidad cognitiva de docentes y estudiantes. La utilización adecuada de herramientas informáticas es un recurso invalorable en cuanto a versatilidad y accesibilidad a estos fines, ya que permite que los estilos individuales se vean reflejados en ellas. Esta implementación, que 
sumó enfoques diversos en función de los estilos de las docentes, les permitió a los alumnos un repaso periódico del material presentado que vino a reforzar la fijación del conocimiento como resultado de la propia actividad directa de los alumnos. Es deseable replicar y multiplicar la experiencia aquí presentada de manera de generar espacios compartidos entre todos los docentes de una misma Cátedra. La experiencia en "parejas pedagógicas" resultó promisoria también en el nivel universitario.

\section{REFERENCIAS}

Bekerman, D. y L. Galagovsky, El lenguaje gráfico de la química: una perspectiva para el análisis de errores. Enseñanza de las Ciencias, Número Extra VIII Congreso Internacional sobre Investigación en Didáctica de las Ciencias, Barcelona, 496-501(2009).

Benoit R. y Haugh B., Team Teaching Tips for Foreign Language Teachers. The Internet TESL Journal, VII (10) (2001). http://iteslj.org/Techniques/Benoit-TeamTeaching.html Acceso: 21 de Noviembre (2010).

Calzadilla M., Aprendizaje colaborativo y tecnologías de la información y la comunicación Revista Iberoamericana de Educación 1-10 (2001) http://www.unabvirtual.edu.co/cpe/docs/ calzadilla_maria_eugenia_Trabajo_colaborativo.pdf Acceso: 27 de noviembre (2010).

Cukierman, U., J. Rozenhauz, y H. Santángelo, Tecnológia educativa, Recursos, modelos y metodologías, $1^{\text {a }}$ Edición, 97, Pearson, Buenos Aires, Argentina (2009).

Delors, J. La Educación encierra un Tesoro Informe a la UNESCO de la Comisión Internacional sobre la Educación para el Siglo XXI. Ediciones UNESCO (1997).

Felder, R. y L.. Silverman, Learning and teaching styles in engineering education, Engineering Education Journal 78 (7), 674-681(1988).

Feldman D. y M. Palamidessi, Programación de la enseñanza en la universidad en Problemas y enfoques, $1^{\circ}$, Colección Universidad y Educación Serie Formación Docente № 1, pp 28-29, Pcia. de Buenos Aires, Argentina (2001).

Menéndez, O, P. de Paco y J. Parrón. Aplicación de metodologías de enseñanza cooperativa en la titulación de Ingeniería Técnica de Telecomunicación, Revista de Formación e Innovación Educativa Universitaria. 2(1), 31-37 (2009).

Pombo, I. y N. Costa Avaliação De Impacte De Cursos De Mestrado Nas Práticas Profissionais De Professores De Ciências - Exemplos De Boas Práticas. Investigações em Ensino de Ciências 14(1), 83-99 (2009). http://www.if.ufrgs.br/ienci/artigos/Artigo_ID208/v14_n1_a2009.pdf. Acceso: 27 de noviembre (2010).

Ribas, M. y M. Vital dos Santos, Desenvolvimento Profissional Dos Formadores De Professores De Ciências No Contexto Da Inovação: Subsídios Teóricos E Metodológicos Para Análise De Um Programa. Investigações em Ensino de Ciências - V15(1), pp. 201-218, (2010).

Suárez, C., E. Burgos e I. Molina, Los estilos pedagógicos y su impacto en el aprendizaje de los alumnos, Civilizar, 5, 181-191 (2003)

Zhang K. y C. Yan-Hua, Participate in the experiment teaching under informatization environment, Agriculture Network Information, Doi: CNKI:SUN:JSJN.0.2008-04-040 (2008) 